\title{
THE EFFECTS OF INJECTING INTRA ARTICULAR PLATELET-RICH PLASMA ON PAIN SCORES IN KNEE JOINT OSTEOARTHRITIS
}

\author{
Khaleel Ahmad, Liaqat Ali*, Muhammad Aasam Masoom Maan** \\ Nawaz Shareef Medical College, Gujrat Pakistan, *Fauji Foundation Hospital, Rawalpindi Pakistan, **Faisalabad Medical University, Faisalabad Pakistan
}

\begin{abstract}
Objective: To determine the effects of intra articular platelet rich plasma (PRP) injection on level of pain of knee joint in patients with knee osteoarthritis.

Study Design: Prospective observational study.

Place and Duration of Study: Combined Military Hospital Rawalpindi, from Aug 2018 to Jan 2019.

Methodology: Forty patients with pain due to knee osteoarthritis for intra-articular injection were included in this observational clinical trial after informed consent and fulfilling inclusion criteria. After measuring pain by numerical rating scale, patients received intra articular platelet rich plasma $(10 \mathrm{ml})$ therapy in affected knee under fluoroscopy. Using the Numerical rating scale level of pain was evaluated and recorded for each patient at different intervals.

Results: There was dramatic improvement in pain score after first and second injection of platelet rich plasma. There was $33.33 \%$ improvement at $3^{\text {rd }}$ week, $54.32 \%$ at $6^{\text {th }}$ week and $71.60 \%$ at $6^{\text {th }}$ month.

Conclusion: Platelet rich plasma is safe, cost effective and minimally invasive in reducing pain scores and functional limitation in knee osteoarthritis.
\end{abstract}

Keywords: Fluoroscopy, knee, osteoarthritis, platelet-rich plasma.

This is an Open Access article distributed under the terms of the Creative Commons Attribution License (https://creativecommons.org/licenses/by-nc/4.0/), which permits unrestricted use, distribution, and reproduction in any medium, provided the original work is properly cited.

\section{INTRODUCTION}

Musculoskeletal system is one of the systems of human body which undergoes physiological changes with aging. Meanwhile, wear and tear of human tissues such as bone and muscle is another observed phenomenon of old age ${ }^{1}$. Lumber spine and neck of femur are most common sites affected in elderly by pathological process of Osteopenia.

Osteoarthritis (OA) is most common form of arthritis affecting people worldwide. This degenerative joint disease causes destruction ofarticular cartilage and synovial joints in elderly people. OA is characterized by joint pain, swelling, tenderness, stiffness, movement restriction, bone spurs and joint deformities. With advancement in diagnostics, a large number of aging population is diagnosed with OA leading to high impact on human quality of life ${ }^{2}$.

OA is global economic burden on society due to increasing incidence and high economic cost. Currently multiple treatment strategies are in practice for OA. These are three fold i.e. non pharmacological e.g physical and rehabilitation therapy, exercise, massage and occupational therapy, pharmacological and surgical therapies in terms of joint replacement ${ }^{3}$. Recently it has been observed that medicines with regenerating ability

Correspondence: Dr Khaleel Ahmad, Department of Pain Medicine, Combined Military Hospital Rawalpindi Pakistan

Received: 13 Jul 2020; revised received: 10 Aug 2020; accepted: 19 Aug 2020 of human tissues are helpful in relieving OA symptoms and reversing tissue pathology. These drugs have shown promising results not only in reducing replacement surgeries but also played important role in palliation. Platelet rich plasma (PRP) is one ofleading drug among this category ${ }^{4}$.

PRP has evolved as one of the advanced treatment options in treating early knee $\mathrm{OA}^{5}$. It is obtained from autologous blood and is enriched with platelets and variety of growth factors. PRP accelerates healing of cartilage morphology in damaged joint by enhancing synovial cell proliferation and differentiation. Platelet rich plasma is one of regeneration therapy that reduces pain and promotes healing of degenerated tissues $^{6}$. PRP trials have been conducted for the treatment of osteoarthritis, including femoral head osteonecrosis, cartilage trauma and rheumatoid arthritis with promising outcome ${ }^{7}$. It has already been claimed by Lee et al that PRP has beneficial role in treatment of rheumatoid arthritis with minimal side effects by inhibiting inflammatory process in involved joint synovial fluid ${ }^{8}$. Many growth factors, bioactive molecules and cytokines in extracted PRP are an integral part of innovative treatment protocol ${ }^{9}$. Moreover, efficiency of PRP was established by Sadabad et al for the treatment of knee osteoarthritis ${ }^{10}$.

The rationale of this study was to make an assessment on the justification of the approach taken towards the use of intra-articular injection of platelet rich plas- 
ma for the treatment of OA pain and define a new modality in chronic pain management for OA pain.

\section{METHODOLOGY}

This prospective observational study was conducted at Combined Military Hospital Rawalpindi, from August 2018 to January 2019 after approval from ethics committee/institutional review board (IRB) ltr no: $07 / 04 / 62 / 20$.

Sample size of 40 patients (20 patients in each group) is calculated by using $80 \%$ power of the test, $5 \%$ level of significance and incidence of patients with requirement of dexmeditomidine in first six hours postoperatively will be $40 \%$.

$p 1=$ probability population $1=40 \%$

$p 2=$ probability population $2=40 \%$

$\mathrm{Z}=$ confidence level $=1.48$

$\mathrm{n}=40$

Sampling technique: non-probability convenience sampling.

After admission of patient in pain clinic, knee examination was conducted and grading of OA was done by Kellgren-Lawrence classification based on radiological features. After written informed consent patients with grade 1-3 were included in study. Patients with previous knee surgery, rheumatoid arthritis, hemophilia and on anticoagulants were excluded.

Standard monitoring including blood pressure, heart rate, pulse oximetry and electrocardiogram was attached to every patient in pain clinic. Under aseptic conditions $30 \mathrm{ml}$ of autologous blood was taken in pre dedicated centrifuge kits for obtaining platelet rich plasma. This sample was centrifuged for 20 minutes at $3200 \mathrm{rpm}$. The collected plasma was centrifuged again for 5 minutes at 1,500 rpm speed. Thus final $10 \mathrm{~mL}$ of injectate solution, rich in platelets was prepared for intra-articular knee injection.

In procedure room, under sterile conditions, after administration of local anesthetics, $23 \mathrm{G}$ spinal needle was inserted in knee joint. $1 \mathrm{ml}$ of dye (Omnipaque 350) was given and needle position confirmed under fluoroscopy. After confirmation of needle position $10 \mathrm{ml}$ of PRP solution was given under aseptic measures. Patients were kept under observation for 1 hour after procedure. Paracetamol tablets were advised if they feel post procedural pain.

Levels of knee pain were evaluated by numerical rating scale (fig-1) and recorded for every patient at specified time point, immediately before the first injection, after 3 weeks prior to second injection, at 6 weeks prior to third injection and 6 months later.

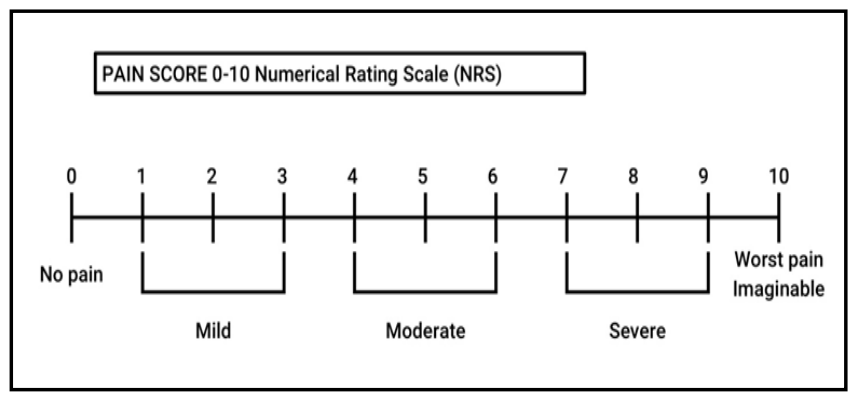

Figure-1: Numerical rating scale.

Data were extracted using mean \pm SD for quantitative variables and frequency and percentage for qualitative ones. Analysis was made using prism graph pad software version 7 . For quantitative variables, to measure significance $\mathrm{t}$-test was applied. The $p$-value $\leq 0.05$ was considered statistically significant.

\section{RESULTS}

In total, 40 patients were included which received PRP intra articular injection at different time intervals. The demographic characteristics of the patients are shown in table-I. The patients in the inclusion criteria did not differ significantly in terms of age and sex.

Figure-1 showed the numeric pain scale (NRS) used in this study and value of NRS at different times intervals was shown in table-I. The value of pain score in patients included in the study according to the NRS criteria was shown in table-II. The patients suffered

Table-I: Value of NRS at different times intervals.

\begin{tabular}{l|c|c|c|c|c}
\hline NRS before PRP & 8.1 & 0.79829 & 40 & 0.26688 & \\
\hline $\begin{array}{l}\text { NRS before 2nd } \\
\text { session at 3 wks }\end{array}$ & 5.4 & 0.82846 & 40 & 0.33569 & 3.8984 \\
\hline $\begin{array}{l}\text { NRS before 3rd } \\
\text { session at 6 wks }\end{array}$ & 3.7 & 0.55431 & 40 & 0.27429 & 1.115 \\
\hline NRS at 6th month & 2.3 & 0.62715 & 40 & 0.44388 & 3.231 \\
\hline
\end{tabular}

Table-II: Numeric rating pain scale at different intervals.

\begin{tabular}{l|c|c|c|c}
\hline $\begin{array}{l}\text { Total number } \\
\text { of patients }\end{array}$ & $\begin{array}{c}\text { before } \\
\text { PRP }\end{array}$ & $\begin{array}{c}\text { at 3rd } \\
\text { Week }\end{array}$ & $\begin{array}{c}\text { at } 6 \text { th } \\
\text { week }\end{array}$ & $\begin{array}{c}\text { at } \mathbf{6} \\
\text { months }\end{array}$ \\
\hline 40 & 8.1 & 5.4 & 3.7 & 2.3 \\
\hline$p$-value & 0.26688 & 0.33569 & 0.27429 & 0.44388 \\
\hline
\end{tabular}

from knee arthritis showed severe pain which was 8.1 on NRS criteria. However, after intra articular injection of PRP at different time intervals there was significant decrease in pain score. At third week of PRP injection, NRS score was 5.4 which was significantly less than NRS value before PRP injection ( $p=0.33$ ). In the comparison of NRS between different time intervals of PRP injection, there was significantly less NRS score at 6th 
week interval $(p=3.7)$ as compared to 3rd week interval $(p=0.33)$. However, more pronounced effect was observed at $6^{\text {th }}$ month of PRP injection which showed the significant reduction in pain score (2.3) as compared to $3^{\text {rd }}$ and $6^{\text {th }}$ week interval $(p=0.44)$ indicating the prolonged effect of PRP after intra articular knee injection in OA as shown in fig-2.

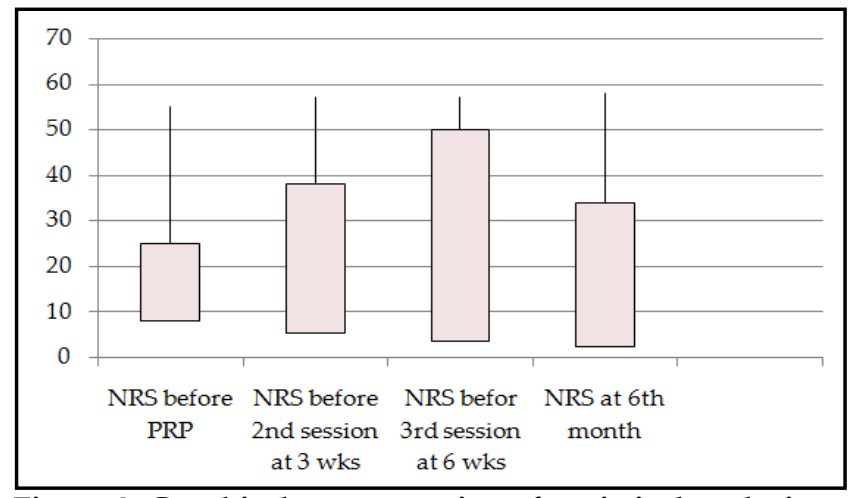

Figure-2: Graphical representation of statistical analysis.

\section{DISCUSSION}

Degenerative joint diseases are sequel of aging. Knee joint OA is one of common disease among them. Previously for knee OA multiple treatment modalities including invasiveand non-invasive have been tried. Primary treatment for knee OA is analgesics proceeded by Physical and rehabilitation therapy. Intra-articular injection of hyaluronic acid and corticosteroids have been tried as second line of treatment. Platelet rich plasma (PRP), prolotherapy (PRL), and radiofrequency ablation (RFA) are next step in treatment modality ${ }^{11}$. Surgical intervention in form of joint replacement is last resort but with its own complications in this age group. Recent studies have shown significant improvements in patient-reported symptoms with results favoring PRP over Hyaluronic acid ${ }^{12}$. Many studies have ascertain beneficial role of PRP therapy in minimizing painand symptoms of $\mathrm{OA}$.

Each $\mathrm{ml}$ of platelet rich plasma solution contain approximately 1-2 million platelets and variety of growth factors. Upon activation platelets release multiple substances including growth factors and cytokines. These substances are involved in increased proliferation and differentiation of chondrocytes. Furthermore, PRP also inhibits NF-KB pathway for its anti-inflammatory effects ${ }^{13}$.

This study proved that platelet rich plasma therapy reduced pain and improved functional capacity of knee OA patients for up to 6 months. Although the treated patients observed decrease in pain in prog- ressive fashion up to six weeks after therapy, however maximum benefit was seen up to 6 months in pain relief and functional ability. Platelet rich plasma therapyis safe and cost effective in short term symptomatic relief in knee osteoarthritis ${ }^{14}$.The degree of knee OA is an important factor to determine the efficacy of platelet rich plasma injection and duration of symptoms relief. PRP therapy has shown good results in young population at early stage of disease ${ }^{15}$.

Duymus et al observed that patients with mild to moderate knee OA experienced better pain relief and pain free daily activities up to 12 months, when treated with Platelet rich plasma as compared to Hyaluronic acid and Ozone injections ${ }^{16}$.

Intra-articular injection of PRP results in reduction of significant degenerative articular inflammatory changes. Himanshu et al concluded that Mean synovial fluid cartilage oligomeric matrix protein concentration was dramatically less in platelet rich plasma therapy treated patients. They also observed decreased synovial vascularity and synovitis in these patients at three and six months interval ${ }^{17}$. Wen et al compared effects of platelet rich plasma, Hyaluronic acid and saline in affected OA knees. They come up with conclusion that int-raarticular Platelet rich plasma injection may be more beneficial in pain relief and functional improvement in symptomatic patients at 1 year post injection interval18. Investigators observed a remarkable difference between platelet rich plasma and hyaluronic acid groups of patients in terms of Western Ontario and McMaster Universities Osteoarthritis Index (WOMAC) scores. Patients with PRP therapy showed better functional scores. In a systematic review, the results were favorable for platelet rich plasma intervention as compared to NSAIDs, hyaluronic acid and corticosteroids in symptomatic knee OA up to 12 months ${ }^{19}$. Deficiency of growth hormone is another important factor in determining the severity of articular cartilage pathologyin Knee $\mathrm{OA}^{20}$. This study proved that platelet rich plasma therapy is more useful in osteoarthritic knee patients whose condition has not been improved by conventional treatment.

Lack of morphological assessment of knee joint, small sample size and limited time frame are some limitations of this study.

\section{CONCLUSION}

Platelet rich plasma is simple, cost effective and less invasive therapy for treatment of knee joint degenerative lesions. Platelet rich plasma therapy also has important role in improving functional outcome and 
alleviating pain of knee OA. This study, proved beneficial role of Platelet rich plasma in decreasing pain and functional limitations in knee OA patients up to 6 months of follow-up. Platelet rich plasma intervention for knee joint OA has minimal side effects.

For better understanding of mechanism of action and measure outcome of platelet rich plasma, well designed randomized controlled trials are essential.

\section{CONFLICT OF INTEREST}

This study has no conflict of interest to be declared by any author.

\section{REFERENCES}

1. JafariNasabian P, Inglis JE, Reilly W, Kelly OJ, Ilich JZ. Aging human body: changes in bone, muscle and body fat with consequent changes in nutrient intake. J Endocrinol 2017; 234(1): 37-51.

2. Ma YW, Jiang DL, Zhang D, Wang XB, Yu XT. Radial extracorporeal shock wave therapy in a person with advanced osteonecrosis of the femoral head. Am J Phys Med Rehabil 2016; 95(9): e133-39.

3. Das G, Dutta D, Roy C, Das S. Can Platelet-rich Plasma Injection Help Avoid Replacement Surgery in Osteoarthritis of the Knee? J Recent Adv Pain 2019; 5(1): 13-15.

4. Krsticevic M, Jeric M, Dosenovic S, Jelicic KA, Puljak L. Proliferative injection therapy for osteoarthritis: a systematic review. Int Orthop 2017; 41(4): 671-79.

5. Ramaswamy RSH, Reddy R, Babu NC, Ashok GN. Stem-cell therapy and plateletrich plasma in regenerative medicines: A review on pros and cons of the technologies. J Oral Maxillofac Pathol 2018; 22(3): 367-74.

6. Chouhan DK, Dhillon MS, Patel S, Bansal T, Bhatia A, Kanwat $\mathrm{H}$. Multiple platelet-rich plasma injections versus single plateletrich plasma injection in early osteoarthritis of the knee. Am J Sports Med 2019; 47(10): 2300-07.

7. Lee GW, Park KS, Kim DY, Lee YM, Eshnazarov KE, Yoon TR. Results of total hip arthroplasty after core decompression with tantalum rod for osteonecrosis of the femoral head. Clin Orthop Surg 2016; 8(1): 38- 44.

8. Fu CJ, Sun JB, Bi ZG, Wang XM, Yang CL. Evaluation of platelet rich plasma and fibrin matrix to assist in healing and repair of rotator cuff injuries: A systematic review and meta analysis. Clin Rehabil 2017; 31(2): 158-172.

9. Vannini F, Di Matteo B, Filardo G. Platelet-rich plasma to treat ankle cartilage pathology - from translational potential to clinical evidence: a systematic review. J Exp Orthop 2015; 2(1): 2-10.

10. Sadabad HN, Behzadifar M, Arasteh F, Behzadifar M, Dehghan HR. Efficacy of platelet rich plasma versus hyaluronic acid for treatment of knee osteoarthritis: A systematic review and meta analysis. Electr Phys 2016; 8(3): 2115-22.

11. Smyth NA, Haleem AM, Ross KA, Hannon CP, Murawski CD, Do HT, et al. Platelet rich plasma may improve osteochondral donor site healing in a rabbit model. Cartilage 2016; 7(1): 104-11.

12. Cole BJ , Karas V , Hussey K, Pilz K, Fortier LA. Hyaluronic acid versus platelet-rich plasma: a prospective, double-blind randomized controlled trial. Am J Sports Med 2017; 45(2): 339-46.

13. Lai LP, Stitik TP, Foye PM, Georgy JS, Patibanda V, Chen B. Use of platelet-rich plasma in intra-articular knee injections for osteoarthritis: a systematic review. PMR 2015; 7(6): 637-48.

14. Bennell KL, HunterDJ, Paterson KL. Platelet-rich plasma for the management of hip and knee osteoarthritis. Curr Rheumatol Rep 2017; 19(5): 24-32.

15. Milants C, Bruyere O, Kaux JF. Responders to platelet-rich plasma in osteoarthritis: a technical analysis. Biomed Res Int 2017; 2017: 7538604.

16. Duymus TM, Mutlu S, Dernek B, Komur B, Aydogmus S, Kesiktas FN. Choice of intra-articular injection in treatment of knee osteoarthritis: platelet-rich plasma, hyaluronic acid or ozone options. Knee Surg Sports Traumatol Arthrosc 2017; 25(2): 485-92.

17. Kanwat H, Singh DM. The effect of intra-articular allogenic platelet rich plasma in dunkin-hartley guinea pig model of knee osteoarthritis: Muscles Ligaments Tendons J 2017; 7(3): 426-34.

18. Dai WL, Zhou AG, Zhang H, Zhang J. Efficacy of platelet-rich plasma in the treatment of knee osteoarthritis: a meta-analysis of randomized controlled trials. Arthscopy. J Arthoscopic Related Surg 2017; 33(3): 659-70.

19. Kanchanatawan W, Arirachakaran A, Chaijenkij K, Prasathaporn $\mathrm{N}$, Boonard M, Piyapittayanun $\mathrm{P}$, et al. Short-term outcomes of platelet-rich plasma injection for treatment of osteoarthritis of the knee. Knee Surg Sports Traumatol Arthrosc 2015; 24(5): 1665-77.

20. Wei FY, Lee JK, Wei L, Qu F, Zhang JZ. Correlation of insulinlike growth factor 1 and osteoarthritic cartilage degradation: a spontaneous osteoarthritis in guinea-pig. Eur Rev Med Pharmacol Sci 2017; 21(20): 4493-500. 